Modern Pharmacology-Toxicology Volume 8 Receptors and Mechanism of Action of Steroid Hormones Part II Edited by Jorge R. Pasqualini. (Pp. 736; illustrated; Sw.fr. 130.) Marcel Dekker: New York. 1977.

This is the second in a two volume publication on the receptors and mode of action of steroid hormones. Part I dealt with the general concept of intracellular cytoplasmic proteins as receptors for steroid hormones with the isolation of these receptor proteins and, finally, with oestrogen and androgens as examples. The present volume continues with the glucocorticoids and mineralocorticoids followed by the applicability of these ideas in assessing the degree of hormone dependence of breast cancer and, finally, the regulatory role of steroid hormone receptors in the brain.

The five chapters are essentially separate essays with little evidence of overall editorial supervision. For example, chapter 8 gives 40 pages of references and full titles, whereas the remaining chapters content themselves with author and journal, and a corresponding reduction in space.

Nevertheless, the subject matter is highly topical, and in breast cancer an important essay gives a clear description of our knowledge in these areas with extensive references to the relevant literature up to $1974 / 75$. The book is essentially aimed, if not at the specialist, at least at those who have special reason for interest in these steroid hormcnes and to those the volume can be recommended.

J. S. GILLESPIE

\section{Chemical Pharmacology of the Synapse} By D. J. Triggle and C. R. Triggle. (Pp. 654; illustrated; $£ 20.00$.) Academic Press: London. 1976.

New discoveries in the chemical process of neurotransmission have been a fecund source both of greater understanding in biology and important advances in medical treatment. In recent years the rate of advance particularly in central neurotransmission has been such that it is difficult for those working in the field, and impossible for those in allied sciences, to keep up. This formidable text bridges this gap most ably. It describes, at a cellular and molecular level, the nature of the synapse, the regulation of both pre- and post-synaptic elements and the events which follow the release of transmitter. Throughout, the emphasis is on the fundamental nature of these events and this, together with extensive references to the original literature, does not make for light reading. Nevertheless, the reward is there for those who apply themselves and there will be few, whether pharmacologists, biochemists, physiologists, or clinicians, who, after reading it, will not see their own problems in a new light.

The book is well produced; there is a clarity and unity of style in spite of the double authorship. It should probably be regarded as a reference book rather than one to be read through, and as such can be confidently recommended to all biological scientists and clinicians with an interest in neural function.

J. S. GILLESPIE

Neurological Development in Infancy By Bert Touwen. (Pp. 150; illustrated; f5.00.) William Heinemann Medical Books: London/J. B. Lippincott Co.: Philadelphia. 1976.

This book is the result of a detailed and systematic study of neurological development in 51 normal infants. Each infant was examined at home by the author every four weeks until the stage of independent walking. At each visit Dr Touwen recorded the developmental stage of each of an extensive battery of test items. By simple graphical display and more complex statistical methods, he now presents the mode of development of each of these items and their interrelationships. Items which did not develop after birth or which showed a very rapid developmental course in early infancy are presumed to depend on early maturing neurological systems and such test items are suitable for inclusion in neurological examinations in infancy. A large number of other items showed a clear developmental sequence with an initial and final change during the study period and an appreciable developmental range. The details of many of these sequences are of great interest to neurologists. Of particular note is the development of voluntary grasping, in the course of which Dr Touwen has noted an upsetting, but fortunately brief, phase of index finger pointing or $\frac{O}{Z}$ 'tipping' in which the infant cannot $\mathbb{D}$ pick up a small object, manifest between the stages of inferior pincer and mature pincer grasping. This tipping is thought to signal the linking of cortical $O$ and non-cortical mechanisms which have been maturing independently. $\frac{\rho}{5}$ Other studies of the relationships between the developing items bring some $\vec{z}$ surprises-for instance, the dissolution of the palmar grasp reflex is not a straightforward prerequisite for the $\overrightarrow{0}$ development of voluntary grasping. Re- $\frac{\square}{O}$ garding the assessment of development, $\frac{\bar{\sigma}}{\bar{s}}$. this book does not of course contain a $\frac{\nabla}{\sigma}$ set of normative data but it points to $\varrho$ the pitfalls of assessment and demon- $\stackrel{\varpi}{\%}$ strates soundly the variability between $\vec{\circ}$ infants and between aspects of develop- ? ment in the same infant, and shows $\vec{\omega}$ convincingly that single examinations $\underset{\sigma}{\omega}$ are inadequate for neurological or $\overline{\bar{z}}$ developmental assessment in infancy. As a basis for further understanding and research into infant developmeract it is essential reading. J. B. P. STEPHENSON

Brain Dysfunction in Infantile Febrife Convulsions Volume 2 Edited by Mary A. B. Brazier and Flavio Cocean (Pp. 384; illustrated; \$33.00.) Rav Press: New York. 1976.

It is a special pleasure to review the proceedings of the IBRO symposium on $\vec{\theta}$ fever and the developing brain which. was held in Toronto in the fall of 1975 o as a satellite to the First International Congress of Child Neurology. Unlike many large international gatherings it was an exciting and human meeting, $\frac{\mathscr{D}}{\phi}$ and this well-knit book reflects and $\varnothing$ focu'es that excitement. The problem $\vec{\overrightarrow{ }}$ posed is why millions of the world's 3 children develop febrile convulsions with fever, and why certain of them have devastating neurological sequelae; the aim is the prevention of the latter, $\overrightarrow{\vec{z}}$ if not the former. Opening chapters illuminate cerebral microdevelopment, and the development of monoaminergic, 3 . cholinergic, and prostaglandin systems. Thermoregulation and mechanisms of $₹$ fever production (and prevention) are $\mathrm{O}$ discussed in detail. On the clinical side, the pathology of febrile convulsions $\frac{D}{0}$ (Meldrum), seizure duration in the genesis of neurological sequelae $N$ (Aicardi and Chevrie), and the ante- or 